\title{
Camera Motions Improve the Sensation of Walking in Virtual Environments
}

\author{
Anatole Lécuyer $^{1}$ \\ SIAMES Project \\ IRISA/INRIA
}

\author{
Jean-Marie Burkhardt ${ }^{2}$ \\ EIFFEL Project \\ University of Paris 5/INRIA
}

\author{
Jean-Marie Henaff $^{3}$ \\ SIAMES Project \\ IRISA/University of Rennes 1
}

\author{
Stéphane Donikian ${ }^{4}$ \\ SIAMES Project \\ IRISA/CNRS
}

\section{AbStract}

This paper investigates the use of camera motions, in order to improve the sensation of walking in a Virtual Environment. A simple model of camera motion is first proposed. This model uses: (1) oscillating motions for the position of the camera, and (2) a compensation motion which changes the orientation of the camera and simulate oculomotor compensation to keep a constant focal point when walking.

Then we describe two experiments which were conducted to study the characteristics of our model and the preference of the users in terms of sensation of walking. The first experiment compared the use of oscillating camera motions along the three directions of space. The oscillating motions were all preferred to the control condition (i.e. a linear motion, as if the user was driving a car). Furthermore, the participants preferred oscillating motions along the vertical axis, compared with the two other directions of space. The second experiment was focused on the use of a compensation motion. It showed that on average participants preferred a compensated motion during the walk, as compared with a motion with a constant orientation of the camera. These results are consistent with the way our body and eyes move naturally when walking in real life.

Taken together, our results suggest that camera motions can considerably improve the sensation of walking in virtual environments. Camera motions could be further introduced in numerous applications of virtual reality in which the simulation of walking is important, such as: architectural visits, training simulations, or videogames.

CR Categories: H.5.1 [Information Interfaces and Presentation]: Multimedia Information Systems - Artificial, augmented, and virtual realities; H.5.2 [Information Interfaces and Presentation]: User Interfaces - Input devices and strategies, Interaction styles, User-centered design; H.1.2 [Information Systems]: User/Machine Systems - Human factors.

Keywords: camera motion, walking, viewpoint, self-motion, sensation.

\footnotetext{
1e-mail: anatole.lecuyer@irisa.fr

2e-mail: jean-marie.burkhardt@inria.fr

3 e-mail: jean-marie.henaff@irisa.fr

e-mail: stephane.donikian@irisa.fr
}

\section{INTRODUCTION}

Navigation is one of the most fundamental tasks of $3 \mathrm{D}$ interaction in Virtual Reality (VR) [7] [27]. Walking in a Virtual Environment (VE) is obviously necessary in numerous applications of VR such as for training (simulation of technical procedures, military infantry operations), design (virtual visit of urban or architectural projects) or simply for fun (videogames).

The quest for a higher "realism" of virtual environments has led designers of VR systems to perpetually find new ways of increasing the sensation of walking of the user. For instance, researchers have proposed sophisticated locomotion interfaces to provide the users with their missing vestibular and proprioceptive cues when walking in a VE [10]. Today, devices such as actuated simulation platforms can be used to displace physically the body of the user, consistently with the visual motion [25]. However, the use of such interfaces still remains expensive and complex.

In order to improve the sensation of walking in a virtual world at lower expenses, videogames introduced slight dynamic changes of the user's viewpoint, i.e. motions of the camera used to display the VE. However, the use of camera motions in videogames remains limited and needs to be properly evaluated. We do not know today if the presence of such camera motions really improves the sensation of walking, and if it does not distort the user's perception of the virtual environment. As for today, there is a need for two kinds of investigation about the use of camera motions to improve the sensation of walking in virtual environments:

1. Development of new models of camera motion in order to improve the sensation of walking in VE;

2. Evaluation of camera motions in terms of both subjective preference and perception of the properties of VE.

In this paper, we provide partial answers to these two issues. We propose a user-centered approach which combines technical concepts and experimental evaluations. First we introduce a new model of camera motion in order to improve the sensation of walking in VE. Then, two experiments are conducted to evaluate our assumptions. The first experiment focuses on the direction of oscillating camera motions that the user would prefer. The second experiment investigates the use of a compensation motion which maintains a fixed focal point for the camera. The paper ends with a conclusion and a description of potential perspectives.

\section{RELATED WORK}

In real life, humans can feel the sensation of walking through different sensory modalities [5]. Self-motion information comes first from the vestibular sense, the proprioceptive sense and the efferent copy of the muscular command [13]. In addition, walking sensation can be provided by other cues such as audition or even smell. Vision is also a major actor in the perception of selfmotion. For instance, the "vection" phenomenon is a strong illusion of motion induced by an optical flow [12]. 
The mutual influences of the sensory modalities involved in walking remain largely unknown. Harris et al. [14] examined the contribution of visual and non-visual cues on the perception of self-motion. They showed that the perceived distance of selfmotion can be estimated from a passive visual flow. However, a passive physical motion turned out to be also a particularly important cue: "not only does it evoke an exaggerated sensation of motion, but it also tends to dominate other cues" [14].

Researchers have proposed numerous tools to simulate physical and vestibular cues when walking in a virtual environment. Slater et al. [26] presented a technique to move through an immersive $\mathrm{VE}$ across distances greater than the physical limitations imposed by tracking devices. For this aim, the user simply "walks in place" and then moves in the direction of his/her gaze. Lécuyer et al. [19] have shown that a haptic (force) feedback sent in the dominant hand of the user could be used to improve the perception of selfmotion in virtual reality.

Today, many force feedback joysticks [3] or force-feedback wheels are used to increase the sensations of the gamers and provide them with haptic effects when driving a car or any other virtual ships. Numerous locomotion interfaces were also developed to enable the user of a virtual reality system to walk physically in the virtual environment while remaining at the same location in the real environment. Examples of recent and promising locomotion interfaces are the Circulafloor [16] or the omni-directional treadmills [24]. Simulation platforms can also be used to reproduce accelerations and displace physically the user in the real environment. These devices are used to improve the immersion of the user in flight or driving simulators [25]. Last, a direct stimulation of the vestibular system was recently proposed by Maeda et al. [20]. This device sends a current between the ears through a helmet to make the user feel disequilibrium toward left or right.

Another approach consists in using the visual feedback of the virtual environment. Lécuyer et al. [17] have shown indeed that it is possible to use visual information to substitute for haptic sensations. This effect was called "pseudo-haptic feedback" [17] and was used to simulate many haptic properties such as friction or stiffness. In videogames, the viewpoint of the gamer can be slightly dynamically modified when walking in the virtual world. The resulting motions of camera are used to provide different types of information. They can be used to inform about the mental or physical state of the avatar (illness, fatigue, etc). They can also be used to increase the sensation of walking. In "Half-Life" [4], the camera motion consists in an oscillating motion along the vertical axis with a small amplitude. In "Project IGI" [2] the camera motion combines an oscillating vertical motion with small variations of the advance speed. The game "Turok 2: Seeds of Evil" [1] provides probably one of the most elaborated camera motion for First-Person Shooting videogames. It is made of a vertical oscillation, an horizontal displacement and even a slight roll effect. However, the amplitude of oscillation of these camera motions remains very small for the moment. This could be due to the fact that these games are fighting games in which the user needs a very high precision. The gamer would not want to be disturbed by parasite movements. For this reason, videogames make use of other means to increase the sensation of walking. One of these efficient tricks consists in moving the weapon which is manipulated by the avatar, at the bottom of the screen. In this case, complex 3D oscillating motions can be applied to the weapon as well as automatic animations (weapon recharging, cleaning, etc). Other special effects such as sound can also be used. In many videogames (or other virtual reality applications [18]), sounds of footstep (and their echoes) are "realistic" cues that can be used as indicator of the speed of motion.
Last, camera motions have been studied and used for other purposes than to improve sensation of walking in VE. Otaduy and Lin [22] developed an algorithm to reposition automatically the user viewpoint for haptic visualization. A lot of techniques have been developed to control the virtual camera and make the user focus on certain objects of the VE [15] [21]. Bowman et al. [8] categorized and evaluated different travel techniques in VE which are associated with different camera motions. Several famous metaphors for viewpoint motion and control in VE have been developed such as the "flying", "eyeball-in-hand" or "World-inMiniature" [8]. Last, Turner et al. [28] described a "physicallybased interactive camera motion control" that uses the laws of classical mechanics to achieve realistic motions of camera in response to force data coming from $3 \mathrm{D}$ input devices.

\section{Model of Camera Motion Used}

In this section we propose a simple model of camera motion to improve the sensation of walking in VE, which is based on observations and on related work in the field of analysis of motion and animation of virtual characters.

\subsection{Use of Oscillating Motions}

Related works in analysis of motion and animation of virtual humans stressed the predominance of oscillating motions during the walking motion [6] [9] [23]. For instance, Boulic et al. [6] gave detailed trajectories of walking for the animation of virtual characters. The kinematical motion of the human thorax was described by two cumulative sine motions along the vertical and lateral axes [6]. The authors described also the modification of the advance speed of the human thorax during walk by the adjunction of another sine variation [6].

We propose to use these oscillating motions to define our model of camera motion. Figure 1 displays the oscillating motions that we apply to the position of the camera, assuming a constant advance speed.

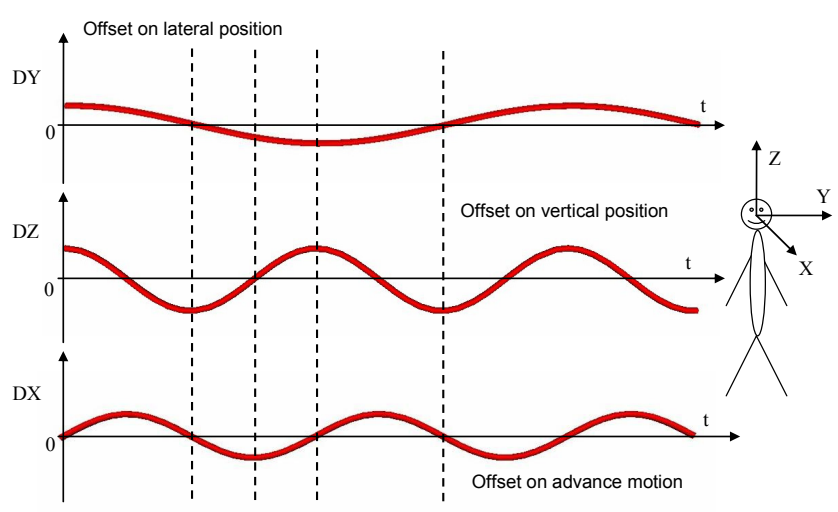

Figure $1-$ (a) Oscillating motion of the camera along the lateral $(\mathrm{Y})$ axis, (b) Oscillating motion of the camera along the vertical $(Z)$ axis, (c) Oscillation on the advance motion i.e. along the $(X)$ axis.

Assuming a constant linear advance speed $\mathrm{V}_{0}$ along the axis of motion (X), the simplified 3D coordinates of the camera are provided by the set of equations (1). In these equations, $t$ is the time, $\mathrm{T}$ is the period of the walking motion, $\mathrm{DX}, \mathrm{DY}$ and $\mathrm{DZ}$ are the offsets in position in the three directions of space, and Ax, Ay and $\mathrm{Az}$ are the three amplitudes of the oscillating motions. 


$$
\left\{\begin{array}{l}
\mathrm{DX}=\mathrm{Ax} * \cos \left(2 \mathrm{Pi} / \mathrm{T}^{*} \mathrm{t}+\mathrm{Pi} / 2\right) \\
\mathrm{DY}=\mathrm{Ay} * \cos (\mathrm{Pi} / \mathrm{T} * \mathrm{t}+\mathrm{Pi} / 2) \\
\mathrm{DZ}=\mathrm{Az} * \cos (2 \mathrm{Pi} / \mathrm{T} * \mathrm{t})
\end{array}\right.
$$

The period $(\mathrm{T})$ is related to the stride length $(\mathrm{L})$ and to the advance speed $\left(\mathrm{V}_{0}\right)$ as follows:

$$
\mathrm{T}=2 * \frac{\mathrm{L}}{\mathrm{Vo}}
$$

Figure 2 displays the resulting trajectory of the camera in 3D. On this Figure, each cone corresponds to one position of the camera at each (constant) time step of the simulation.

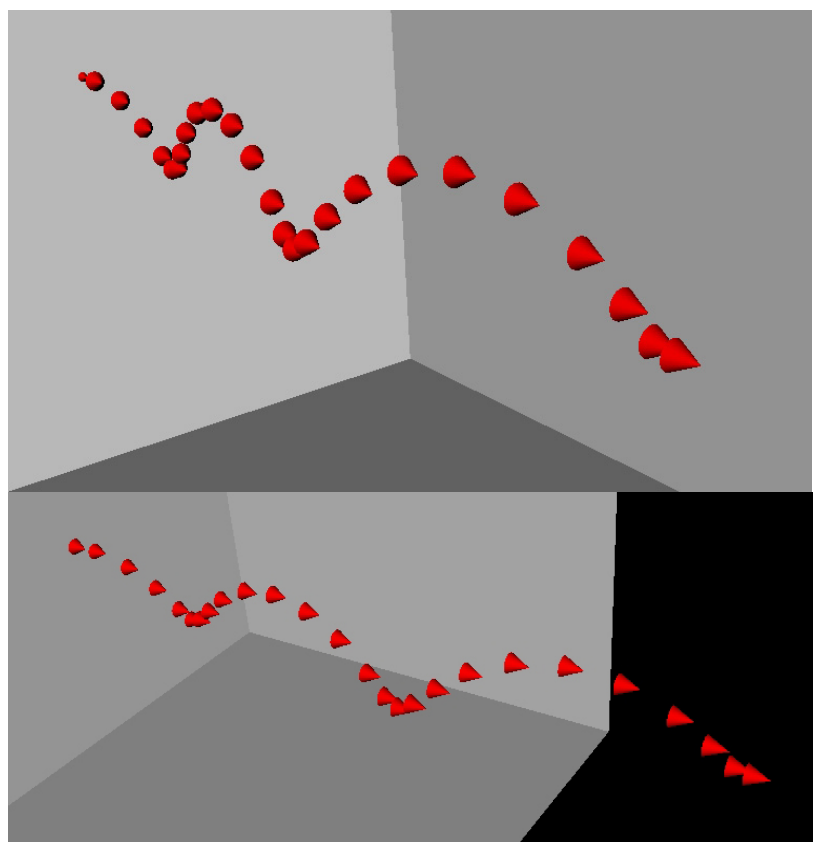

Figure 2 - Two different views of the 3D trajectory used for the camera motion $(A x=A y=A z=10 \mathrm{~cm})$.

\subsection{Use of a Compensation Motion}

In the few examples of camera motion which were used in videogames, the camera keeps a constant orientation (see Figure 3a). The visual feedback resulting from such a motion corresponds to the visual flow provided by a camera posed on the top of the head when walking. This visual feedback is very different from what happens when walking in real life. In reality, our gaze is always focused on an object i.e. on a temporary constant focal point. When we walk in a real environment, our eyes compensate for the motion of our body so to maintain the focalization on this point or object. Numerous studies in biomechanics and analysis of motion have stressed the importance of the eyes for steering locomotion [5] [11]. As Alain Berthoz puts it: "I go where I look, and not I look where I go" [5].

Therefore, we propose to incorporate in our model changes in the orientation of the camera in order to simulate the gaze compensation motion (oculomotor compensation). This compensation motion is illustrated on Figure $3 b$. (a) Constant orientation of the camera

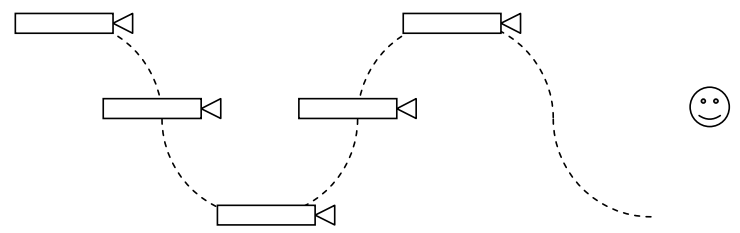

(b) Changing orientation of the camera to maintain focalization

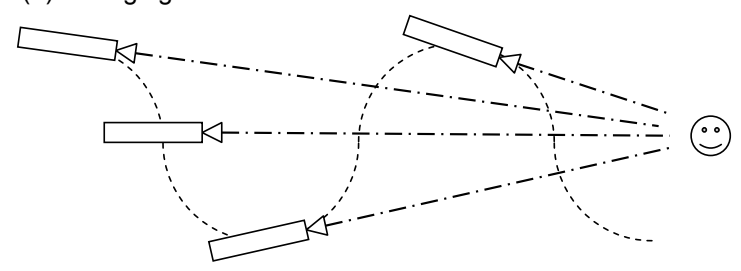

Figure 3- (a) Constant orientation of the camera, (b) Changes in orientation of the camera to maintain a fixed focal point

Assuming that the VR system knows where (i.e. at which position $\mathrm{O}$ ) is located the object that the user is looking at, the change in orientation of the camera vector $(\vec{v})$ corresponds to a simple rotation. This rotation aims at aligning the camera vector $(\vec{v})$ and $(\overrightarrow{C O})$, with $\mathrm{C}$ being the current position of the camera.

The main issue of this model consists in knowing the position $(\mathrm{O})$ of the object that the user is looking at. There are several paradigms which could be used to know this location. A first possibility consists in using an eye-tracking system to measure precisely the gaze of the user in the virtual environment [29]. A second paradigm could be used in applications like First-Person Shooting games. In such applications, the gamer uses and manipulates a target which is displayed at the center of the screen (a visor), in order to shoot at enemies. This target (or any other cursor manipulated by the user) could be used as an indicator of where the user is watching. For instance, the system could use the depth information (Z-Buffer) at the center of the visor to compute the position of the focal point. With such an implementation, the camera motion would not disturb the user anymore when shooting at enemies at the level of the visor.

\subsection{Final Model of Camera Motion}

The final model of camera motion that we propose is the combination of the two aforementioned techniques:

1. The oscillating motion for the position of the camera: the combination of the three sine motions displayed on Figure 1, which corresponds to the complex motion illustrated on Figure 2;

2. The compensation motion to change the orientation of the camera and maintain a focalization on the virtual object that the user is watching, as displayed on Figure $3 b$.

\subsection{Implementation of the Model for the Experimental Evaluation}

Two experiments were designed to evaluate step by step the properties of our model. The first experiment was focused on the use of oscillating motions. The second experiment was focused on the use of a compensation motion.

After some preliminary testing, the stride length (L) was set to $0.8 \mathrm{~m}$ and the advance speed $\left(\mathrm{V}_{0}\right)$ was set to $1 \mathrm{~m} / \mathrm{sec}$ in the two 
experiments. According to Equation 2, the period (T) was equal to $1.6 \mathrm{~s}$. The initial coordinates of the user's eyes $\left(\mathrm{X}_{0} ; \mathrm{Y}_{0}\right)$ corresponded to his/her initial position in the virtual environment. The initial altitude $\left(\mathrm{Z}_{0}\right)$ was set to $1.75 \mathrm{~m}$.

We used a basic implementation of the compensation motion for the purpose of the second experiment. In this simplified implementation, the user was explicitly asked to look at one virtual object of the VE (a virtual ball). This virtual ball was located in front of the user and displayed at the centre of the screen (see Figure 5a). When the user walked in the VE, the virtual ball was also moved with the same linear advance speed so to stay perpetually at the same distance in front of him/her. During the walking motion, in addition to the oscillating motions, the camera rotated and kept on orienting itself in the direction of the centre of the virtual ball. The scheme was thus a bit different from what is displayed on Figure $3 \mathrm{~b}$. Since the virtual object was moved with the same translational motion as the user, the change in orientation of the camera was indeed periodical.

\section{EXPERIMENT 1: INVESTIGATING THE USE OF OSCILlaATING CAMERA Motions}

The first experiment was focused on the use of oscillating camera motions to generate a better sensation of walking in VE. It was meant to identify the preferential axis for an oscillating camera motion. This experiment was expected to provide useful indications to better determine the values of parameters Ax, Ay and Az used in Equation 1.

In this experiment, participants were exposed to pairs of successive passive visual motions. These visual motions were generated by the simulation of a displacement of a camera along a linear trajectory in a virtual environment. After each pair, the participants had to indicate which of the two motions they preferred in terms of sensation of walking. Four conditions were compared. The first condition was a control condition using the linear displacement only. The three other conditions used an additional oscillating camera motion with only one direction of oscillation (X, $\mathrm{Y}$ or Z) superimposed to the linear displacement.

\subsection{Participants}

Twelve participants aged between 21 and 33 (mean=28) passed this experiment. There were 9 men and 3 women. All participants were right-handed and had normal or corrected vision. None of them were familiar with the proposed technique.

\subsection{Experimental Plan}

The participants tested 120 pairs resulting from: six (2 by 2) combinations of four motion Conditions (Control, M1, M2 and M3), tested 20 times.

- Control corresponded to a basic linear motion in a virtual environment (displayed Figure 4). In this condition, the camera was displaced along a linear trajectory (as if the user was driving a car) with a constant speed $(\mathrm{Vo}=1 \mathrm{~m} / \mathrm{s})$.

- M1 corresponded to the additional use of an oscillating camera motion along the vertical axis only $(\mathrm{Ax}=0 ; \mathrm{Ay}=0 ; \mathrm{Az}=0.1)$.

- M2 corresponded to the additional use of an oscillating camera motion along the lateral axis only $(\mathrm{Ax}=0 ; \mathrm{Ay}=0.1 ; \mathrm{Az}=0)$.

- M3 corresponded to the additional use of an oscillation on the advance motion only $(\mathrm{Ax}=0.1 ; \mathrm{Ay}=0 ; \mathrm{Az}=0)$.
The oscillating motions of M1, M2 and M3 were all superimposed to the linear motion of the camera (i.e. superimposed to the Control motion). All motions (M1, M2, M3, and Control) had the same initial and final positions in the virtual environment (see Figure 4).

Half of the pairs of passive motions presented two conditions in one order, while the other half of pairs presented the two same conditions in the opposite order. Order of presentation between the 120 pairs was randomized for each participant within the test session. The overall experiment lasted 40 minutes.

\subsection{Procedure}

Participants were seated $30 \mathrm{~cm}$ in front of a 17 ' computer screen. They had their line of sight aligned with the center of the screen. We used a monoscopic rendering, with a frame rate of $50 \mathrm{~Hz}$.

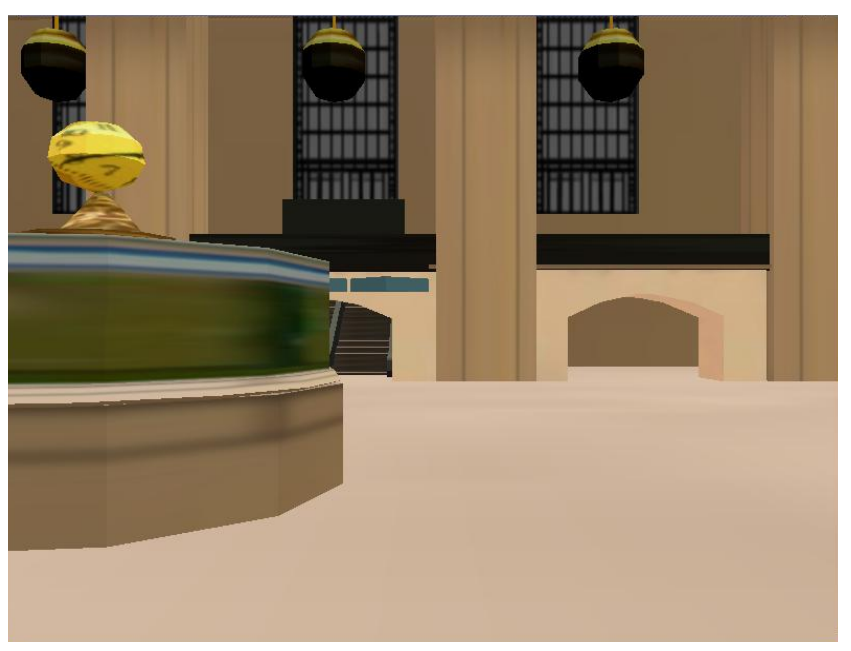

Figure 4 - Experiment 1: Screenshot of the virtual environment used.

Each trial began with a first passive visual navigation under one of the four aforementioned conditions (Control, M1, M2, or M3). The first visual navigation lasted $5 \mathrm{~s}$ after which a blue panel was displayed for $2 \mathrm{~s}$ on the entire screen. Then, the second passive navigation was launched with the same duration, same initial and final positions but with a different condition i.e. with a second camera motion. At the end of the second exposure a blue panel was displayed again for $2 \mathrm{~s}$ and the participant had to enter his/her preference between the two sequences of passive navigation.

The instruction was to choose which navigation was better in terms of "sensation of walking". The participants could enter their answer using the "1" and "2" keys of the keyboard (respectively for the $1^{\text {st }}$ and $2^{\text {nd }}$ navigation). When they validated their choices (using the space bar), the next trial was automatically launched.

At the end of the experiment, the participants could try again the 4 different motions separately as many times as they wanted. They were asked to fill in a subjective questionnaire. In this questionnaire, they had to rank the 4 different conditions (the 4 different motions) according to their preference in terms of sensation of walking.

\subsection{Data Collected}

The choices of the participants were automatically recorded for each pair of motions they were exposed to. 
The questionnaires filled by the participants at the end of the experiment were also collected, and the final rankings of the participants were analyzed.

\subsection{Results}

The Table 1 summarizes the results found for Experiment 1 concerning the choice of the participants when they had to compare the pairs of motion. The camera motion based on an oscillation along the vertical axis (M1) was the most appreciated condition, since it was chosen on average $71.7 \%$ of the time when present in a trial. The Control condition (linear motion) was the less favored motion since it was chosen only $33.5 \%$ of the time when present within one trial. The preferences for the two other oscillating motions M2 (45.6\%) and M3 (49.3\%) were located between M1 and Control.

We performed an Analysis of Variance (ANOVA) on the choices of participants. ANOVA showed a significant main effect of the Condition $(\mathrm{F}(3,44)=3.760, \mathrm{p}<.018)$. The $\mathrm{M} 1$ condition appeared to be significantly preferred to the Control condition (Fisher PLSD test, $\mathrm{p}<.002$ ) and to the M2 condition (Fisher PLSD test, $\mathrm{p}<.030$ ). The comparison between $\mathrm{M} 1$ and $\mathrm{M} 3$ failed to reach significance (Fisher PLSD test, $\mathrm{p}<.061$ n.s.).

\begin{tabular}{|l|l|l|l|l|}
\hline Condition : & Control & M1 & M2 & M3 \\
\hline$\%$ of selection : & $33.5 \%$ & $71.7 \%$ & $45.6 \%$ & $49.3 \%$ \\
\hline Standard deviation & $26.2 \%$ & $32.4 \%$ & $26.4 \%$ & $28.4 \%$ \\
\hline
\end{tabular}

Table 1 - Choice of participants: percentage of selection (and standard deviations) for each condition when it was present in a trial.

The Table 2 displays the ranking of participants, as computed from the subjective questionnaires. We analyzed the frequencies related to the way participants ranked the four conditions. There was a strong relationship between the Condition and the participants' ranking (V2 Cramer=.18), meaning that marked preferences were expressed by the participants. The test on this relationship was significant (Khi2 $=25.33$, dof $=9, \mathrm{p}<.0026$ ).

Data revealed once again that $\mathrm{M} 1$ was preferred by participants, since 8 participants out of 12 (i.e. $67 \%$ ) have placed it in first position. The Control motion was ranked in third position by 7 participants and in fourth by 3 participants. This implies that the Control condition was negatively evaluated by 10 participants out of 12 (i.e. 84\%). Conditions M3 and M2 are located between Control and M1 again, but M3 seems to be slightly better appreciated than M2.

\begin{tabular}{|c|c|c|c|c|}
\hline Rank & Control & M1 & M2 & M3 \\
\hline 1st (best) & 1 & 8 & 1 & 2 \\
\hline $2^{\text {nd }}$ & 1 & 2 & 4 & 5 \\
\hline 3rd & 7 & 0 & 2 & 3 \\
\hline $4^{\text {th }}$ (worst) & 3 & 2 & 5 & 2 \\
\hline
\end{tabular}

Table 2 - Ranking of the participants for the four motions.

\subsection{Discussion and Conclusion}

This first experiment showed clearly that oscillating camera motions were appreciated by the participants for improving sensation of walking. The oscillating motion along the vertical axis (M1) was strongly preferred, when compared to a lateral oscillating motion (M2) or to an oscillation applied to the advance motion (M3). The linear-only movement of the camera (Control condition) was clearly the less appreciated condition. These results seem consistent with related work in analysis of motion and biomechanics which stressed the importance of vertical oscillating motions in the human walk [6].

\section{EXPERIMENT 2: INVESTIGATING THE USE OF A COMPENSATION MOTION}

The second experiment was focused on the second component of our model i.e. the compensation motion. This second experiment investigated the preference of the users when provided with the compensation motion and without.

The task was here again to select the preferred motion in terms of sensation of walking among a pair of two passive visual navigations. Three conditions were tested. They corresponded to three different camera motions: a complex camera motion with a compensation motion, a complex camera motion without a compensation motion (i.e. with a constant orientation), a Control condition i.e. with a linear motion.

\subsection{Participants}

Ten new participants (who did not participate in experiment 1), aged between 20 and 46 (mean=29), took part in this experiment. There were 6 men and 4 women. One person was left handed. All participants had normal or corrected vision. None of them were familiar with the proposed techniques.

\subsection{Experimental Plan}

The participants tested 90 pairs resulting from the 3 (2 by 2) combinations of 3 Conditions (Control, Mv, Mv_comp), with 3 different trajectories in the virtual environment (T1, T2, T3) and 10 trials per pair of comparison.

- The Control motion corresponded to a basic linear motion in the virtual environment, as described in Experiment 1.

- Mv corresponded to the use of a complex camera motion superimposed to the linear motion of the camera (i.e. superimposed to the Control motion). Mv used the combination of the three oscillating motions displayed on Figure 1. As a consequence of Experiment 1, the vertical oscillation was amplified compared to the two other oscillations. The three parameters $(\mathrm{Ax}, \mathrm{Ay}, \mathrm{Az})$ were set to: $\mathrm{Ax}=0.05 ; \mathrm{Ay}=0.05$; and $\mathrm{Az}=0.1$.

- Mv_comp corresponded to the use of the same complex camera motion as Mv, but superimposed with the compensation motion described in section 2.4. A virtual ball was displayed at the center of the screen in front of the user, at a $10 \mathrm{~m}$ distance (see Figure 5). As already mentioned, this ball was moving linearly with the same speed as the user $\left(\mathrm{V}_{0}\right)$. The camera kept on rotating and changing its orientation to stay focused on this object. 

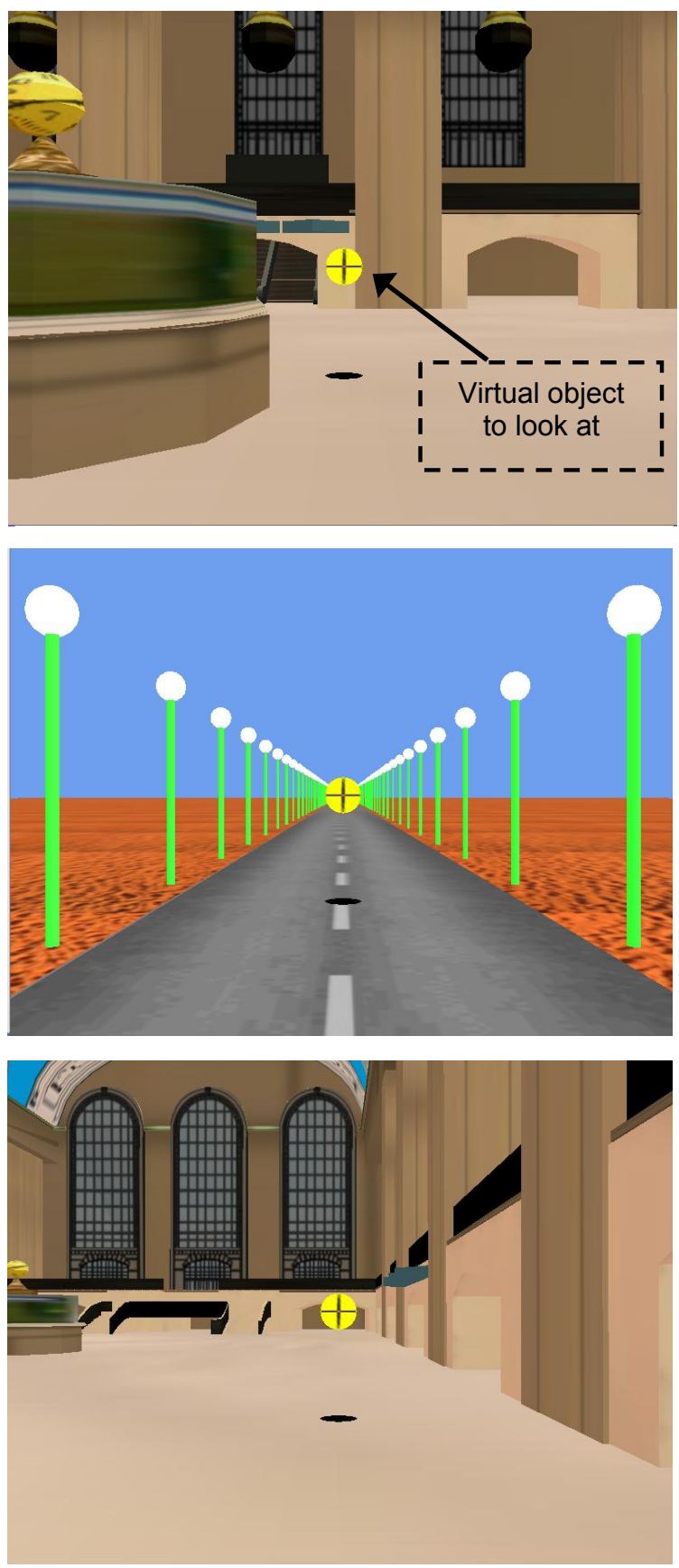

Figure 5 - Experiment 2: Screenshots of the three possible trajectories $(\mathrm{T} 1, \mathrm{~T} 2, \mathrm{~T} 3)$.

All motions Mv, Mv_comp and Control were achieved with the same initial and final positions in the virtual environment. The virtual ball was displayed in front of the user in all conditions (Control, Mv, Mv_comp), in order to ensure a visual consistency between all three experimental conditions.

Half of the pairs of motions presented two conditions in one order, while the other half of pairs presented the two same conditions but in the opposite order. Order of presentation between the 90 pairs was randomized for each participant within the test session.

The same materials as in experiment 1 were used, except that three different trajectories (T1, T2 and T3) were used (see Figure 5).
The same procedure was used as in experiment 1 . However, the participants were here explicitly asked to keep looking at the center of the virtual ball located in front of them (see Figure 5a). They were asked to "always look at the center of the black cross mapped on the ball". The experiment lasted 30 minutes.

\subsection{Collected Data}

For each trial, we recorded the answer of the participant (i.e. the preference for the " $1 \mathrm{st}$ " or " 2 nd" navigation in terms of "sensation of walking").

\subsection{Results}

On average, participants preferred the cases when the compensation motion was present. Indeed, $\mathrm{Mv}$ comp was chosen in $71 \%$ ( $\mathrm{sd}=28 \%$ ) of cases when it was present. It is followed by Mv, which was selected in $56 \%$ of cases $(\mathrm{sd}=17 \%)$. The linear motion (Control) was the less preferred motion with only $21 \%$ of choice $(\mathrm{sd}=19 \%)$.

An ANOVA with repeated measures was performed on the choices of participants within trials. The within-subjects factors were the three experimental conditions (Control, Mv, Mv_comp) and the three different trajectories (T1, T2, T3). The results showed a main significant effect of the condition on the choice of the participants $(\mathrm{F}(2,18)=14.999, \mathrm{p}<.0002)$. As expected, no significant effect of the trajectory was found. A posteriori tests showed that the Control condition was significantly less appreciated than $\mathrm{Mv}$ and Mv_comp (PLSD Fisher, $\mathrm{p}<.002$ ). The difference between conditions $\mathrm{Mv}$ and $\mathrm{Mv}$ comp failed to be highly significant, although better results for Mv_comp were observed. Last, there was a marginally significant two-ways interaction between the experimental condition and the trajectory $(F(4,36)=44.061, p<.03)$.

\subsection{Discussion and Conclusion}

This second experiment confirmed first that a camera motion made of oscillating motions was largely preferred by participants for giving a better sensation of walking in a virtual environment. This time, the camera motion we used was a combination of three sine motions, as described by the set of equations (1). It was a more "realistic" motion than the elementary sine motions used in Experiment 1.

Second, our results suggest that the presence of a compensation motion (change in orientation and fixed focal point) is preferred by the users, although ANOVA test failed to be significant. The failure of the ANOVA could be due to two reasons: first, the relatively small number of participants. Second, it could be due to the current implementation of the compensation motion. The use of a more "natural" paradigm could maybe lead to a higher preference. However, future work (using for example an eye-tracking system) must be done to investigate these assumptions.

\section{General Conclusion and Perspectives}

This paper investigated the use of camera motions to improve the sensation of walking in virtual environments. We first described a model of camera motion which combines oscillating motions with a compensation motion inspired by the way our gaze stays focused on objects when walking. 
This model was evaluated through two experiments. The first experiment investigated the use of oscillating motions. It showed the predominance of vertical oscillations, which were highly preferred by participants. The second experiment used complex camera motions combining oscillating motions in the three directions of space with a compensation motion. This second experiment confirmed first the strong subjective preference of participants for oscillating camera motions when compared to a linear motion (i.e. as if the user was driving a car). Then it showed that on average the presence of the compensation motion was preferred by the participants.

Taken together, our results showed the positive influence of camera motions on the sensation of walking in virtual environments. Camera motions could thus be used in numerous VR applications such as: training simulations, virtual visit of architectural sites, videogames, etc.

Future work. Future work could first investigate other models of camera motion taking into account properties of the human walk. Second, we could study the use of the compensation motion in more "ecological" situations. To improve user-friendliness, we plan to use an eye-tracking system to define the focal point of the virtual camera. Third, we would like to study the influence of such camera motions on the users' perception of properties of virtual environments. For instance, we could evaluate the influence of camera motions on the estimation of distances when walking in a virtual environment. We would also like to study the potential (positive/negative) influence of camera motions on cybersickness. Last, we would like to study the influence of several parameters on our results such as: the level of immersion (use of larger screens e.g., CAVE), the use of active vs. passive navigations, the use of long vs. short exposure, etc.

\section{REFERENCES}

[1] http://www.acclaim.com/

[2] http://www.eidos.com/

[3] http://www.immersion.com/

[4] http://www.sierra.com/

[5] A. Berthoz, «Le sens du Mouvement», Odile Jacob Eds, Paris, 1998.

[6] R. Boulic, N. Magnenat-Thalmann, and D. Thalmann, "A Global Human Walking Model With Real-Time Kinematic Personnification", The Visual Computer: International Journal of Computer Graphics, Vol. 6, pp. 344-358, 1990.

[7] D. Bowman, E. Kruijff, J. LaViola, and I. Poupyrev, "3D User Interfaces: Theory and Practice", Addison-Wesley Eds, 2004

[8] D. Bowman, D. Koller, L.F. Hodges, "Travel in Immersive Virtual Environments: An Evaluation of Viewpoint Motion Control Techniques", Proc. of the Virtual Reality Annual International Symposium, 1997

[9] S.K. Chung, and J.K. Hahn, "Animation of Human Walking in Virtual Environments", Proc. of the Computer Animation, 1999.

[10] D.P. Darken, W.R. Cockayne and D. Carmein, "The omnidirectional treadmill: a locomotion device for virtual worlds", Proc. of the ACM symposium on User Interface Software and Technology, pp. 213-221, 1997.

[11] R. Grasso, P. Prevost, Y.P. Ivanenko, and A. Berthoz, "Eye-head coordination for the steering of locomotion in humans: anticipatory synergy", Neuroscience Letters, Vol. 253, pp. 115-118, 1998.

[12] R. Gurnsey, D. Fleet, and C. Potechin, "Second-order motions contribute to vection", Vision Research, Vol. 38, pp. 2801-2816, 1998.

[13] L.R. Harris, M. Jenkin, and D.C. Zikovitz, "Vestibular cues and virtual environments: choosing the magnitude of the vestibular cue", Proc. of IEEE International Conference on Virtual Reality, pp. 229236, 1999.
[14] L.R. Harris, M. Jenkin, D. Zikovitz, F. Redlick, P. Jaekl, U. Jasiobedzka, H. Jenkin, and R. S. Allison, "Simulating self motion I: cues for the perception of motion", Virtual Reality, Springer-Verlag, Issue 6, Num. 2, pp. 75-85, 2002

[15] S. Hughes, and M. Lewis, "Attentive Navigation for Viewpoint Control in Virtual Environments", Human Factors, Vol. 47, Num. 3, pp. 630-643, 2005.

[16] H. Iwata, H. Yano, H. Fukushima, and H Noma, "CirculaFloor", IEEE Computer Graphics and Applications, Vol. 25, Num. 1, pp. 64-67, 2005

[17] A. Lécuyer, S. Coquillart, A. Kheddar, P. Richard, and P. Coiffet, "Pseudo-Haptic Feedback: Can Isometric Input Devices Simulate Force Feedback?", Proc. of IEEE International Conference on Virtual Reality, 2000.

[18] A. Lécuyer, P. Mobuchon, C. Mégard, J. Perret, C. Andriot, and J.P. Colinot, "HOMERE: a Multimodal System for Visually Impaired People to Explore Virtual Environments", Proc. of IEEE International Conference on Virtual Reality, 2003.

[19] A. Lécuyer, M. Vidal, O. Joly, C. Mégard and A. Berthoz, "Can Haptic Feedback Improve the Perception of Self-Motion in Virtual Reality?", Proc. of Symposium on Haptic Interfaces for Virtual Environment and Teleoperator Systems, 2004.

[20] T. Maeda, H. Ando, T. Amemiya, M. Inami, N. Nagaya, and M. Sugimoto, "Shaking the World: Galvanic Vestibular Stimulation as a Novel Sensation Interface", ACM SIGGRAPH, Emerging Technologies, 2005.

[21] E. Marchand, and N. Courty, "Controlling a camera in a virtual environment", The Visual Computer Journal, Vol. 18, Num. 1, pp. $1-19,2002$

[22] M. Otaduy, and M. Lin, "User-centric viewpoint computation for haptic exploration and manipulation" Proc. of the IEEE Conference on Visualization, pp. 311-318, 2001

[23] J. Pettre, T. Simeon, and J.P. Laumond, "Planning human walk in virtual environments", Proc. of IEEE/RSJ International Conference on Intelligent Robots and Systems, pp. 3048-3053, 2002.

[24] J. Richards, A. Mulavara, and J. Bloomberg, "Postural stability during treadmill locomotion as a function of the visual polarity and rotation of a three-dimensional virtual environment", Presence: Teleoperators and Virtual Environments, Vol. 13, pp. 371-384, 2004

[25] G. Reymond, A. Kemeny, J. Droulez, and A. Berthoz, "Role of lateral acceleration in curve driving: Driver model and experiments on a real vehicle and a driving simulator", Human Factors, Vol. 43 , Num. 3, pp. 483-95, 2001.

[26] M. Slater, M. Usoh, and A. Seed, "Taking steps: the influence of a walking technique on presence in virtual reality", ACM Transactions on Computer-Human Interaction, Vol. 2, Issue 3, pp. 201-219, 1995

[27] D.S. Tan, G.G. Robertson, and M. Czerwinski, "Exploring 3D Navigation: Combining Speed-coupled Flying with Orbiting", Proc. of ACM CHI, 2001

[28] R. Turner, J.F. Balaguer, E. Gobbetti, and D. Thalmann, "Physically-based Interactive Camera Motion Control using 3D Input Devices", Proc. of CGI, pp. 135-145, 1991

[29] S. Zhai, C. Morimoto, and S. Ihde, "Manual and gaze input cascaded (MAGIC) pointing", ACM CHI, 246-253, 1999. 